\title{
Acute Management of Intraperitoneal Extravasation of Total Parenteral Nutrition (TPN) in a Very Preterm Infant: Case Report and Literature Review
}

\author{
Atif Majid \\ Jonathan Barrett \\ Michael P Meyer
}

Neonatal Intensive Care Unit, Middlemore Hospital, Auckland, New Zealand
Correspondence: Michael P Meyer

Neonatal Intensive Care Unit,

Middlemore Hospital, 100 Hospital Road,

Otahuhu, Auckland, New Zealand

Tel +64 21 714 190

Email michael.meyer@middlemore.co.nz
Purpose: Use of umbilical catheters is standard practice in neonatal intensive care units due to ease of insertion and provision of longer-term vascular access. Complications of umbilical venous catheters, including extravasation of total parenteral nutrition (TPN) fluid, are rare but when they occur have high mortality and morbidity. Umbilical venous catheters tend to migrate over time, and their position may change, so a high index of suspicion should be maintained. Our aim was to describe a case with extravasation of parenteral nutrition fluid and review the medical literature.

Patients and Methods: Case report.

Results: The infant was born at 28 weeks' gestation $(1510 \mathrm{~g})$. On day 6 he presented with nonspecific abdominal distension, hypotension, respiratory deterioration, metabolic acidosis and was critically ill. Radiological and ultrasound findings were consistent with TPN ascites due to a malpositioned umbilical venous catheter. Bedside paracentesis without laparotomy was carried out. Despite a stormy course, the infant recovered and the liver injury with conjugated hyperbilirubinemia improved over a period of several months.

Conclusion: Emergency bedside ultrasound and paracentesis with catheter removal may be lifesaving and avoid laparotomy in an already critically unwell preterm infant.

Keywords: total parenteral nutrition, extravasation, ascites, preterm infant umbilical venous catheter, medical management

\section{Introduction}

Siting umbilical venous and arterial catheters is common practice in neonatal intensive care units since $1947 .^{1}$ A correctly placed umbilical venous catheter (UVC) is a convenient way to secure central venous access and has several advantages when compared with peripheral venous cannulation. UVCs enable use of higher osmolality fluid eg total parenteral nutrition (TPN), safe administration of medications such as inotropes and eliminate the stress and pain associated with repeated peripheral venous puncture. ${ }^{2}$ Although it is a relatively easy-to-perform procedure, prior experience with UVC placement shows that ideal catheter tip position (upper border of T8 to lower border of T9 vertebral body) is not achieved in approximately $31.9 \%$ of cases. ${ }^{3}$ Unfortunately, these central vein catheterisations are also associated with a number of complications. These include blood-borne catheter-related infection, blood loss during catheterisation or catheter detachment, air embolism, catheter tip migration, thromboembolic complications, cardiac tamponade, disorders of cardiac rhythm and pericardial or pleural effusion. ${ }^{4-9}$ Although 
thought to be uncommon, complications such as air in the portal system $(20 \%)$, portal venous thrombosis $(6 \%)$ and hepatic lesions $(7 \%)$ have been noted on ultrasound. ${ }^{8}$ Improper position of UVCs can allow leakage of hyperosmolar parenteral nutrition into the liver tissue, with significant damage to the liver parenchyma, hepatic necrosis and ascites. ${ }^{4-6,10}$ Proper positioning of umbilical venous catheters to avoid the above-mentioned catheterassociated complications is paramount. Point-of-care ultrasonography (POCUS) is useful and precise in locating the catheter tip in relation to the portal venous system, inferior vena cava (IVC) and heart when position on the radiograph is uncertain. In a prospective study of 65 extremely low-birthweight infants, only $38 \%$ of catheter tips were in optimal position when its position was re-confirmed by POCUS, with catheter migration reported in up to $50 \%$ during the first week of life. ${ }^{3,11}$ Active surveillance of catheter tip position, by either radiograph or preferably POCUS when expertise exists, is vital to prevent (or aid in early identification of) UVC extravasations. ${ }^{3}$ This was highlighted in a recent study where a 4-day-old, 24-week gestational neonate developed isolated ascites. POCUS revealed a malpositioned $\mathrm{UVC}$, the removal of which resulted in prompt resolution of symptoms and hence prevention of any complications. ${ }^{12}$

\section{Materials and Methods}

\section{Case Presentation} Patient Information

A male infant was delivered vaginally at home following spontaneous preterm labour at 28 weeks and 6 days weighing $1510 \mathrm{~g}$. The pregnancy was complicated by gestational diabetes mellitus. The infant was assigned Apgar scores of 9 and 9 at 1 and $5 \mathrm{~min}$ of age based on the history following an unattended home birth. He was admitted to the neonatal unit at 1 hour of age following ambulance transfer; pulse oximeter saturations were $99 \%$ and core temperature $35.9^{\circ} \mathrm{C}$. Due to respiratory distress, nasal continuous positive airway pressure (CPAP) at $7 \mathrm{~cm} \mathrm{H}_{2} \mathrm{O}$ via Hudson prongs (Teleflex Medical, Wellington, New Zealand) was commenced with room air. He also received caffeine, probiotics and a short course of antibiotics in line with unit guidelines.

A double lumen UVC (Argyle 5Fr, Covidien Auckland, New Zealand) was inserted with the tip just below the diaphragm at the level of T9 thoracic vertebra and the position accepted after discussion with radiology
(Figure 1). Standard TPN solution together with Vitalipid (Biomed, Auckland, New Zealand) was commenced and graded up to $160 \mathrm{~mL} / \mathrm{kg} / \mathrm{d}$ with $3 \mathrm{~g}$ intravenous lipid. Enteral feeds (expressed breast milk) were commenced on day 2 and graded up to $48 \mathrm{~mL} / \mathrm{kg} / \mathrm{d}$ by day 7 and TPN reduced accordingly.

Clinical Findings, Diagnostic Testing and Assessment On day 6 the infant deteriorated when he developed respiratory distress and desaturation events requiring recommencement of CPAP therapy (which had ceased the previous day). He clinically deteriorated further over the next 4-6 hours, developing worsening pallor, hypotension (blood pressure 26/12 $\mathrm{mmHg}$, mean $20 \mathrm{mmHg}$ ), marked abdominal distention, metabolic acidosis $(\mathrm{pH}$ 7.07, $\mathrm{PaCO}_{2} 6.6 \mathrm{kPa}$, base excess -17 ), hyperglycaemia (23.2 $\mathrm{mmol} / \mathrm{L})$ and hyponatraemia $(124 \mathrm{mmol} / \mathrm{L})$. Feeds were stopped and abdominal X-rays ordered. The differential diagnoses at this time included necrotising enterocolitis, sepsis or other acute surgical abdomen such as volvulus. The X-rays showed a picture consistent with fluid in the abdominal cavity, with abdominal distension and a paucity of bowel gas and the catheter tip in the hepatic shadow (Figure 2). Point-of-care abdominal ultrasound confirmed the presence of abdominal fluid, the overall picture being consistent with TPN extravasation/ ascites.

\section{Intervention}

Due to the critical condition of the patient and the fact that paediatric surgical support is not available on site, emergency bedside treatment was performed. The UVC was removed and an ascitic tap performed in the right iliac fossa using a size 17 cannula following infiltration with local anaesthetic; $105 \mathrm{~mL}$ of salmon pink coloured fluid was removed, with marked reduction of abdominal distention. Antibiotics were commenced, and aggressive resuscitation was required including elective intubation, mechanical ventilation and cardiovascular support. An adrenaline bolus was given for hypotension followed by fluid boluses, adrenaline and dopamine infusions and a red blood cell transfusion (15 mL/kg; haemoglobin $108 \mathrm{~g} / \mathrm{L})$. Coagulation screen revealed a normal activated partial thromboplastin time, prothrombin ratio 1.7 and normal fibrinogen level. Blood pressure, blood gases and urine output improved with these measures. Inotropes were gradually weaned over the next 48 hours and the infant extubated to CPAP. The peritoneal aspirate had a glucose 


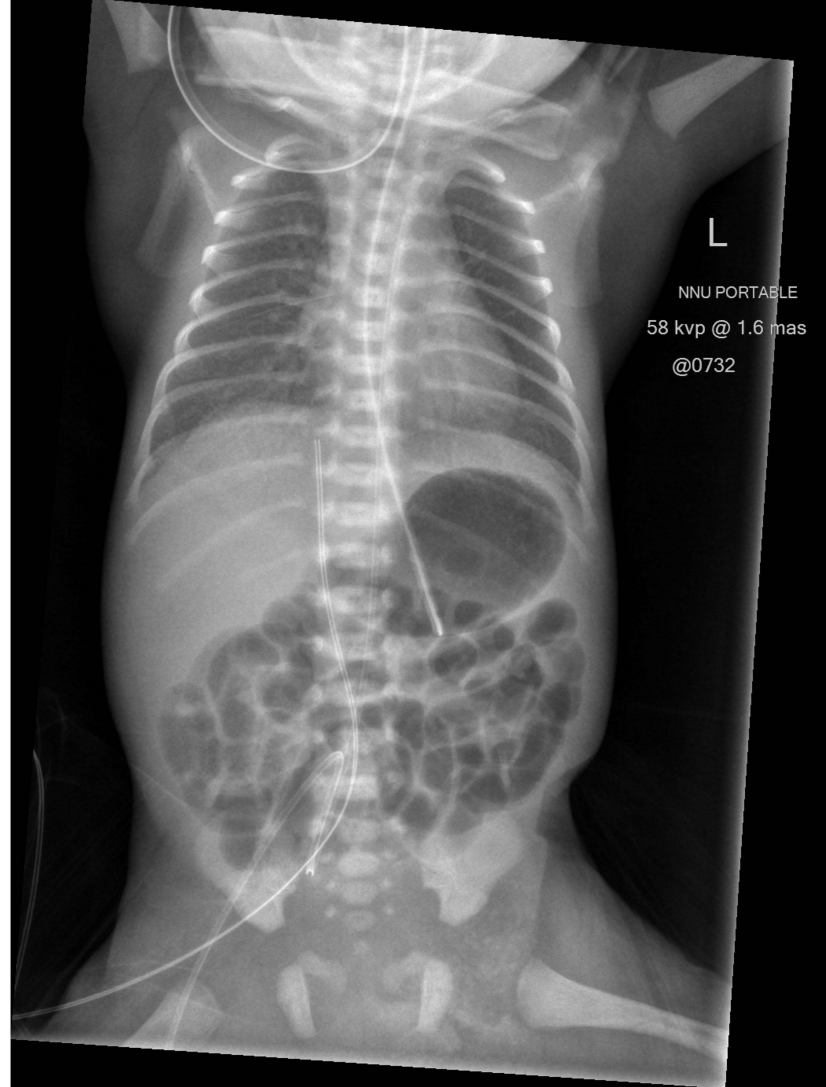

A

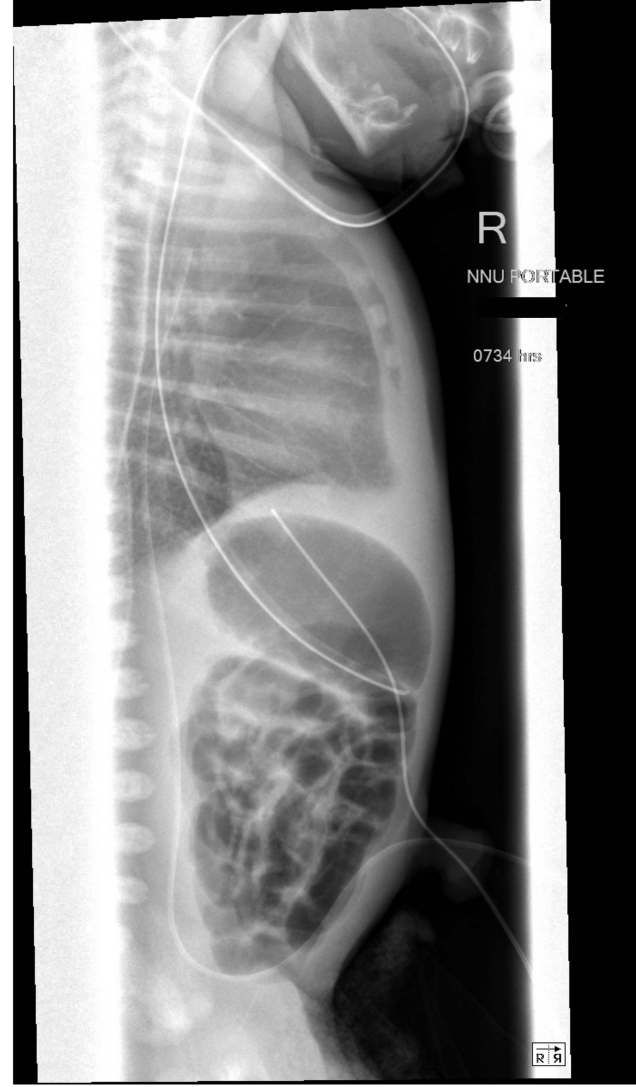

B

Figure I (A and B) Anteroposterior and lateral films showing initial umbilical venous catheter positioning at the level of 9 th thoracic vertebra. The position of the umbilical arterial line was adjusted.

concentration of $56 \mathrm{mmol} / \mathrm{L}$ with protein $<10 \mathrm{~g} / \mathrm{L}$, red blood cells $53 \times 10^{9} / \mathrm{L}$ and white blood cells $2.1 \times 10^{9} / \mathrm{L}$.

\section{Follow-Up and Outcomes}

Subsequent abdominal ultrasound showed a complex fluid collection centred in the right lobe of the liver $(3 \times 4 \times 4 \mathrm{~cm})$ with a hyperechoic avascular rim, the latter probably reflecting liver parenchymal injury (Figure 3). Both kidneys had isoechoic material within the renal pelves associated with minor distention, suspicious for haemorrhage. The liver function tests showed a modest rise in aspartate aminotransferase (AST) levels to $512 \mathrm{U} / \mathrm{L}$, but this resolved within 4 days; there was, likewise a mild transient rise in alanine aminotransferase (ALT) and gamma glutamyl transpeptidase (gamma GT) levels. A conjugated hyperbilirubinemia developed, with a maximum level at 5 weeks after the acute event of $90 \mu \mathrm{mol} / \mathrm{L}$ and total bilirubin level of $109 \mu \mathrm{mol} / \mathrm{L}$. By 3 months, the bilirubin levels were normal. Serial ultrasounds showed resolution of the intrahepatic collection apart from a small, irregular, avascular area of increased echogenicity in the right lobe
(Figure 3). Renal ultrasound a month after the initial scan was normal, although a small pocket of residual intraabdominal free fluid remained.

\section{Discussion}

This very preterm infant suffered acute deterioration due to extravasation of TPN fluid into the liver followed by marked abdominal ascites. Whilst treatment may involve laparotomy for both diagnostic and therapeutic reasons, ${ }^{4,10}$ urgent bedside treatment was required in this case. There have been a number of cases described where this has been successfully carried out, ${ }^{2,11}$ and paracentesis could, therefore, be considered as a less invasive alternative to laparotomy where the diagnosis is likely. There is uncertainty as to removal of the UVC and whether there is an increased risk of bleeding. To our knowledge, there have been no reports of this occurring following bedside removal compared to removal at laparotomy. In one case series, all 4 infants improved following removal at the bedside. ${ }^{11}$ 


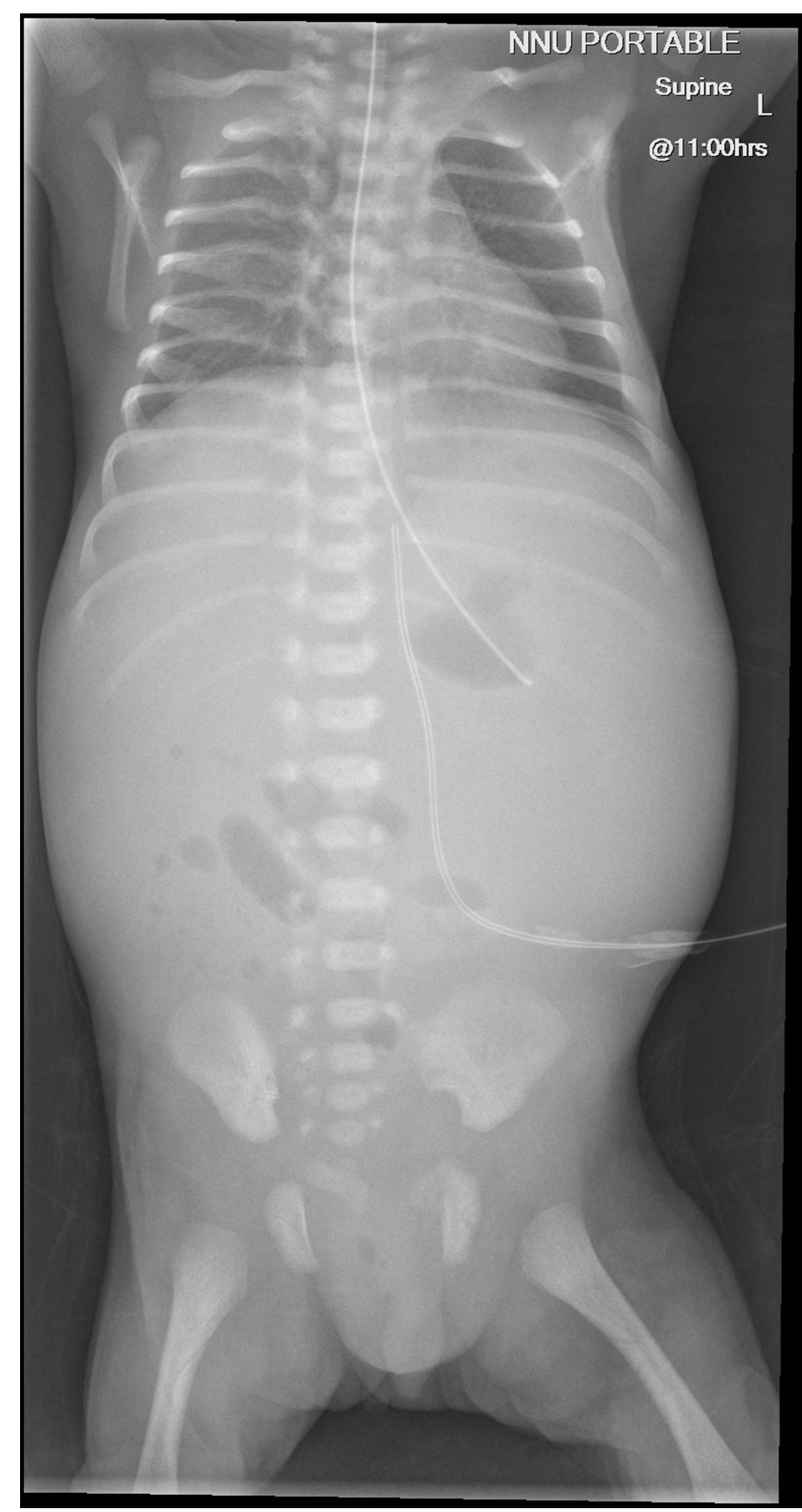

Figure 2 Supine abdominal film showing distension and paucity of abdominal gas (note umbilical venous catheter is now malpositioned and between T9 and TI0).

The insidious nature of the TPN accumulation is suggested by the fact that the volume of fluid removed would have been infused over 24 hours or more, although there was probably blood and exudate in the total.

The appropriate position of UVC is just above or at the level of the diaphragm (T7-T9) between the inferior vena cava and the right atrium after passage through the portal system and ductus venosus. ${ }^{7-10}$ There is a concern of the UVC being positioned in the liver when it is below the diaphragm (T10 or below). The position, although borderline in this case (T9), was accepted following radiology opinion. Repeat abdominal films showed the tip had
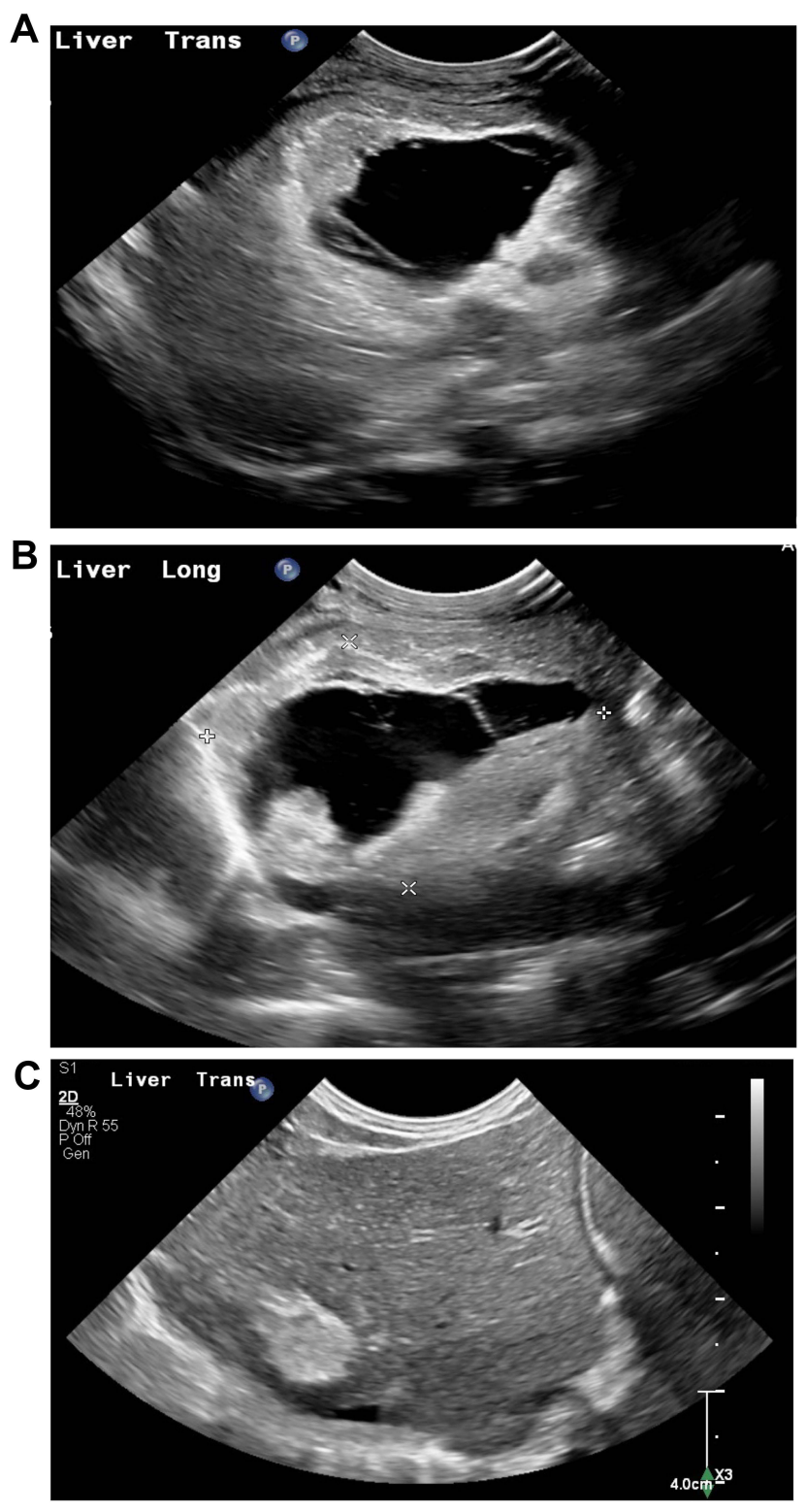

Figure 3 (A) Transverse liver scan showing cavitation and hyperechoic rim. (B) Longitudinal liver scan showing extent of lesion and septation (distance between the markers is $42 \mathrm{~mm}$ ). (C) Transverse liver scan 2 months after the injury showing small residual area (approximately $\mathrm{I} \mathrm{cm}$ in size) in right lobe of liver.

migrated to T11, but whether this occurred de novo or was due to the abdominal distension was unclear. Confirmation of UVC position is usually done radiographically with antero-posterior and lateral films. However, a recent study has shown that localisation of the UVC in preterm infants by X-ray is often imprecise and ultrasound is being increasingly used, ${ }^{11}$ although this is not standard practice in many neonatal units.

As far as the nature of injury is concerned, the catheter tip can directly injure the vessel wall (mechanical microinjury), leading to direct leakage of TPN fluid into the 
peritoneum. ${ }^{13}$ In addition to the mechanical microinjury, hyperosmolar TPN fluid can lead to portal phlebothrombosis and liver necrosis with TPN fluid leakage into necrotic foci, injured sinusoids and small subcapsular veins. ${ }^{2}$ It is likely that fluid collects beneath the liver capsule and then leaks into the peritoneum and may cause a complicated ascites with septae and debris. ${ }^{14}$ This mechanism of catheter malposition leading to vascular injury with subsequent extravasation and ascites has also been previously reported. ${ }^{2}$ Although the hepatic lesion and biochemical anomalies resolved in this case, to date follow-up has been for 6 months, and this is a limitation of the report.

This case highlights the fact that catheter-related complications must be considered whenever there is acute abdominal distension with a UVC in place. Malposition has been recognised as the most common UVC-related complication and the most important risk factor for hepatic extravasation in literature. ${ }^{15}$ Point-of-care ultrasonography is a valuable adjunct investigation to confirm UVC tip placement, especially when information from standard radiographs is uncertain. The clinical spectrum of extravasation injury varies widely from insidious ascites, unexplained change in biochemical parameters to rapid intravascular volume depletion, and potentially catastrophic renal failure. ${ }^{3}$ Paracentesis with catheter removal and aggressive supportive therapy may be lifesaving and potentially avoid the need for laparotomy. Resolution of the liver injury was noted over a period of several months.

\section{Ethical Considerations}

The child's parent has provided informed consent and written approval for publication of this case report. Institutional approval was not required to publish the case details.

\section{Disclosure}

The authors have no conflicts of interest or financial disclosures to declare, and no funding was obtained for the report.

\section{References}

1. Diamond LK. Erythroblastosis fetalis or haemolytic disease of the newborn. Proc R Soc Med. 1947;40(9):546-550.

2. Hargitai B, Toldi G, Marton T, Ramalingam V, Ewer AK, Bedford Russell AR. Pathophysiological mechanism of extravasation via umbilical venous catheters. Pediatr Dev Pathol. 2019;22(4):340343. doi: $10.1177 / 1093526619826714$

3. Edison P, Arunachalam S, Baral V, Bharadwaj S. Varying clinical presentations of umbilical venous catheter extravasation: a case series. J Paediatr Child Health. 2021;57(7):1123-1126. doi:10.1111/ jpc. 15137

4. Pegu S, Murthy P. Ascites with hepatic extravasation of total parenteral nutrition (TPN) secondary to umbilical venous catheter (UVC) malposition in an extremely preterm baby. BMJ Case Rep. 2018;2018:bcr-2018.

5. Shareena I, Khu YS, Cheah FC. Intraperitoneal extravasation of total parental nutrition infusate from an umbilical venous catheter. Singapore Med J. 2008;49(2):e35-e36.

6. Adesanya O, Naqvi M. Term neonate with liver laceration, obstructive uropathy, and ascites-secondary to extravasation of total parenteral nutrition: a complication of malpositioned umbilical venous catheter. Glob Pediatr Health. 2016;3:2333794X1667049. doi:10. 1177/2333794X16670494

7. Yeh J, Boechat M, Smith J, Touma M, Vargas J, Wozniak L. Massive liver mass and parenteral nutrition extravasation secondary to umbilical venous catheter complications. J Clin Neonatol. 2014;3(3):158. doi:10.4103/2249-4847.140404

8. Nakstad B, Naess PA, de Lange C, Schistad O. Complications of umbilical vein catheterization: neonatal total parenteral nutrition ascites after surgical repair of congenital diaphragmatic hernia. $J$ Pediatr Surg. 2002;37(8):1-3. doi:10.1053/jpsu.2002.34497

9. Coley BD, Seguin J, Cordero L, Hogan MJ, Rosenberg E, Reber K. Neonatal total parenteral nutrition ascites from liver erosion by umbilical vein catheters. Pediatr Radiol. 1998;28(12):923. doi:10. 1007/s002470050500

10. Egyepong J, Jain A, Chow P, Godambe S. Parenteral nutrition-ascites with acute renal failure as a complication from an umbilical venous catheter in an extremely low birth weight infant. BMJ Case Rep. 2011;2011(apr21 1):bcr0220113813. doi:10.1136/bcr.02.2011.3813

11. Franta J, Harabor A, Soraisham AS. Ultrasound assessment of umbilical venous catheter migration in preterm infants: a prospective study. Arch Dis Child Fetal Neonatal Ed. 2017;102(3):F251-F255. doi:10.1136/archdischild-2016-311202

12. Rajendran G, Sinha AK. Umbilical venous catheter extravasation diagnosed by point-of-care ultrasound. Arch Dis Childhood. 2021;106(5):549. doi:10.1136/archdischild-2020-320008

13. Schmidt B, Zipursky A. Thrombotic disease in newborn infants. Clin Perinatol. 1984;11(2):461-488. doi:10.1016/S0095-5108(18)30929-1

14. Williams S, Chan AK. Neonatal portal vein thrombosis: diagnosis and management. Semin Fetal Neonatal Med. 2011;16(6):329-339. doi:10.1016/j.siny.2011.08.005

15. Chen HJ, Chao HC, Chiang MC, Chu SM. Hepatic extravasation complicated by umbilical venous catheterization in neonates: a 5year, single-center experience. Pediatr Neonatol. 2020;61(1):16-24. doi:10.1016/j.pedneo.2019.05.004

Research and Reports in Neonatology

Dovepress

Publish your work in this journal

Research and Reports in Neonatology is an international, peerreviewed, open access journal publishing original research, reports, editorials, reviews and commentaries on neonatal health. The manuscript

management system is completely online and includes a very quick and fair peer-review system. Visit http://www.dovepress. com/testimonials.php to read real quotes from published authors. 